\title{
Numerical heat transfer modelling of staggered array impinging jets
}

\author{
A. Ramezanpour ${ }^{1}$, I. Mirzaee ${ }^{2}$, R. Rahmani ${ }^{1}$ \& H. Shirvani ${ }^{1}$ \\ ${ }^{I}$ Faculty of Science and Technology, Anglia Ruskin University, UK \\ ${ }^{2}$ Faculty of Engineering, Urmia University, Iran
}

\begin{abstract}
A numerical study of flow field and heat transfer rate in external flow of a novel heat exchanger (Anglia Ruskin University, 2001) was conducted. The design comprises of confined impinging jets from a staggered bundle of tubes in which the fluid flows in an opposite staggered arrangement array after impingement. The RNG $k-\varepsilon$ model and enhanced wall treatment near wall turbulence modelling was applied to model a three-dimensional computational domain. The accuracy of the model was validated in two- and three-dimensional cases for single impinging jets with available experimental results. The arrangement of the staggered array was fixed $S_{n} / D=2.1$ and $S_{p} / D=1.6$ where $S_{n}$ and $S_{p}$ are the distances between tubes transverse and parallel to fluid flow respectively and $D$ was the hydraulic diameter of tubes. The dimensionless tubes to impinging surface distance $(H / D)$ were in the range of $0.2,0.5,1.0$, and 2.0 and the Reynolds number based on the tubes' hydraulic diameter and average fluid velocity at the exit of tubes in the range of 1000, 5000, and 20000 were studied. The global heat transfer rate on both impinging and confinement plates increased with decreasing of $H / D$ and increasing of Reynolds number; however, the slope of increasing Reynolds number was sharper in low $H / D$ s. The friction factor increased with a decreasing $H / D$ and an increasing of the Reynolds number. The local Nusselt number was studied on both impinging and confinement plates. The temperature contours and velocity vectors are also presented.

Keywords: bundle of impinging jets, jet to jet interaction, $R N G k-\varepsilon$, enhanced wall treatment.
\end{abstract}




\section{Introduction}

The bundle of impinging jets has applications where high heat flux over a small area is needed such as electronic cooling systems and turbine blade cooling. This concept was used to develop a novel compact heat exchanger as external flow in combination with staggered tube bundle (Figure 1).

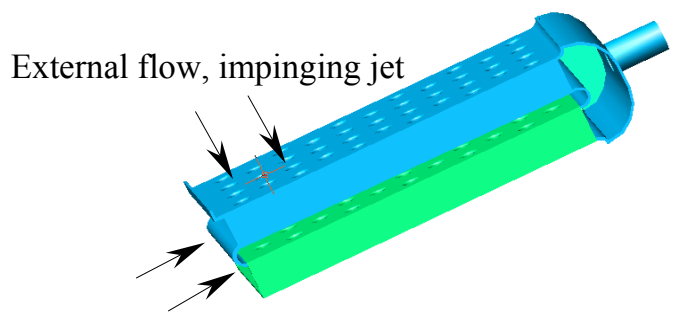

Internal flow, Staggered tube bundle cross flow

Figure 1: Novel compact heat exchanger (Anglia Ruskin University, patent 2001).

Carcasci [1] experimentally studied the single air impinging jet and bundle of jets and their interactions with and without cross flow. For a pair of impinging jets, when the main vortices of two jets interacted with each other, two vortexes were generated in the space between the main vortexes and the bottom flat plate (lower adverse vortexes). The interaction between the main vortices and the topmost flat plate (confinement plate) determined a second series of vortexes (upper adverse vortexes). The experimental heat transfer study of single confined impinging jets, inclined slot nozzle impinging jets, and influence of Prandtl number were carried out by Jung-Yang et al. [2], Beitelmal et al. [3], and ChinYuan and Suresh [4] respectively.

In numerical turbulent studies of impinging jets, although more costly Reynolds stress model due to taking into account turbulence anisotropy effects leads to more satisfactory results than eddy viscosity models (Morris et al. [5]) however satisfactory modelling of the heat transfer rate on impinging surface depends mostly on the near wall turbulence modelling (Craft et al. [6]). The research by Durbin [7] showed that the overestimation of heat transfer rate on the impinging surface by eddy viscosity $k-\varepsilon$ model is due to excessive growth of the turbulent kinetic energy in the stagnation region. Behnia et al. [8] by using $v 2 f$ model studied the influence of confinement, nozzle exit velocity profile and turbulence intensity on the average and local heat transfer of the impinging surface for a single round nozzle. Furthermore Park and Sung [9] obtained satisfactory results by modifying a near wall turbulent model. A study of slot nozzle impinging jets by Ramezanpour et al. [10] used enhanced wall treatment near wall turbulent modelling in Fluent and led to a satisfactory prediction of local heat transfer rate on the impinging surface in either vertical or inclined impinging jets. San and Lai [11] and Su and Chang [12] experimentally studied the bundle of impinging jets for staggered and inline arrays respectively. 
This study is concerned with the numerical flow and heat transfer rate modelling with no equilaterally staggered bundle impinging jet in which flow exits through the opposite geometrical staggered tubes after impingement. The RNG $k-\varepsilon$ model and enhanced wall treatment near wall turbulence modelling is applied.

\section{Numerical modelling}

\subsection{Governing equations}

The steady state continuity, momentum, and energy equations for incompressible flow and constant variables may be presented as:

$$
\begin{gathered}
\frac{\partial u_{i}}{\partial x_{i}}=0 \\
\frac{\partial\left(\rho u_{i} u_{j}+\delta_{i j} p-\tau_{i j}\right)}{\partial x_{j}}=0 \quad \text { where } \tau_{i j}=\mu\left(\frac{\partial u_{i}}{\partial x_{j}}+\frac{\partial u_{j}}{\partial x_{i}}\right) \\
\rho c_{p} u_{i} \frac{\partial T}{\partial x_{i}}=\lambda \frac{\partial^{2} T}{\partial x_{j}^{2}}
\end{gathered}
$$

The turbulent kinematic energy, $k$, and its dissipation rate, $\varepsilon$, in the RNG $k$ - $\varepsilon$ model are modelled as:

$$
\begin{gathered}
\rho \vec{u}_{i} \frac{\partial k}{\partial x_{i}}=\frac{\partial}{\partial x_{j}}\left(\left(\mu+\frac{\mu_{t}}{\operatorname{Pr}_{t}}\right) \frac{\partial k}{\partial x_{j}}\right)+G_{k}-\rho \varepsilon \\
\rho \vec{u}_{i} \frac{\partial \varepsilon}{\partial x_{i}}=\frac{\partial}{\partial x_{j}}\left(\left(\mu+\frac{\mu_{t}}{\operatorname{Pr}_{t}}\right) \frac{\partial \varepsilon}{\partial x_{j}}\right)+C_{1 \varepsilon} \frac{\varepsilon}{k} G_{k}-C_{2 \varepsilon}^{*} \rho \frac{\varepsilon^{2}}{k}
\end{gathered}
$$

where

$$
G_{k}=2 \mu_{t} S_{i j} \frac{\partial u_{j}}{\partial x_{i}} \quad, \quad C_{2 \varepsilon}^{*}=C_{2 \varepsilon}+\frac{C_{\mu} \rho \eta^{3}\left(1-\eta / \eta_{\circ}\right)}{1+\beta \eta^{3}}
$$

and $\eta=S(k / \varepsilon)$ is function of strain rate. The extra term in $\varepsilon$ equation in RNG $k-\varepsilon$ model reduces turbulent eddy viscosity, $\mu_{t}=\rho C_{\mu} k^{2} / \varepsilon$, in high strain rate regions. The constants of the model are:

$$
C_{\mu}=0.0845, C_{1 \varepsilon}=1.42, C_{2 \varepsilon}=1.68, \eta_{0}=4.38, \beta=0.012
$$

The energy equation is solved by approximating the extra temperature fluctuation term as diffusion term with diffusion coefficient equal to the eddy viscosity.

In the near wall region enhanced wall treatment is used in which the inner region $\left(\operatorname{Re}_{y}=\rho y \sqrt{ } k / \mu<200\right)$, momentum and $k$ equations are solved and eddy viscosity as well as dissipation rate are obtained algebraically. In the inner region 
the eddy viscosity and dissipation rate are calculated based on blending function in which its output near the wall leads to the algebraic values of one-equation turbulence model and in boundary of outer region $\left(R e_{y}=200\right)$ leads to the values of RNG $k-\varepsilon$ equation to be solved in the outer region (Fluent [13]).

The temperature and velocity near the wall are obtained by blending the laminar sublayer and turbulent logarithmic laws (Kadar [14]):

$$
\begin{array}{lll}
u^{+}=e^{\Gamma} u_{\text {lam }}^{+}+e^{1 / \Gamma} u_{\text {turb }}^{+} & \\
\frac{d u^{+}}{d y^{+}}=e^{\Gamma} \frac{d u_{\text {lam }}^{+}}{d y^{+}}+e^{1 / \Gamma} \frac{d u_{\text {turb }}^{+}}{d y^{+}} & \text {where } & \Gamma=-\frac{a\left(y^{+}\right)^{4}}{1+b y^{+}} \\
T^{+}=e^{\Pi} T_{\text {lam }}^{+}+e^{1 / \Pi} T_{\text {turb }}^{+} & \text {where } & \Pi=-\frac{a\left(\operatorname{Pr} y^{+}\right)^{4}}{1+b \operatorname{Pr}^{3} y^{+}}
\end{array}
$$

where $a=0.01, b=5.0$, and $\kappa=0.41$ is von Karman constant. The generation term in solving $k$ equation near the wall is obtained using equations (7).

\subsection{Computational domain}

The three dimensional computational domain by symmetrising the external flow in the novel heat exchanger is shown in Fig. 2. The boundaries include tube walls, impinging and confinement surface walls, inlet flow, outlet flow and symmetric boundary all around the domain. The arrangement of tubes is fixed, $S_{p} / D=2.1, S_{n} / D=1.6$. The inlet and outlet tubes have fixed length $L=1 \mathrm{~cm}$, tube diameter $d=1 \mathrm{~cm}$, and $H / D=0.2,0.5,1.0,2.0$ where $D$ is the hydraulic diameter of the tubes. The inlet Reynolds number based on average tube exit velocity and hydraulic diameter of tubes is $R e=1000,5000,20000$.

The velocity in the inlet boundary has linear profile and turbulent intensity and length scale are set to $3 \%$ and $0.01 \mathrm{~cm}$. On the walls no-slip condition is imposed and constant heat flux $100 \mathrm{~W} / \mathrm{m}^{2}$ on impinging surface and $50 \mathrm{~W} / \mathrm{m}^{2}$ on the confinement and tubes are considered. Also to solve the turbulence kinematic energy equation, $\partial k / \partial n=0$ is considered on the walls. In the symmetric boundaries, the gradient of all variables (zero diffusion) as well as velocity component normal to the boundary (zero convection) are set to zero.

Solving the near wall regions, instead of using wall functions, demands fine mesh near to the walls. A 16 layer boundary layer grid with a first grid height of $0.02 \mathrm{~mm}$, growing ration of 1.1 was generated on the impinging and confinement walls. The tetrahedral grid was used to cover the computational domain.

The dimensionless wall parameter in the stagnation point adjacent cell was less than 0.5 in whole range of study (Ramezanpour et al. [10]) slightly less than 1.0 which was recommended (Fluent [13]).

\subsection{Solution parameters}

A finite volume based discretization and segregated and explicit method was used to solve the governing equation. SIMPLE predictive-corrector algorithm 
was used for velocity-pressure coupling and the momentum, energy and turbulence equations were discretized by second order interpolation scheme. The under-relaxation factors were 0.3 for pressure and 0.8 for other parameters. The solution was considered converged when the scaled residual errors were reached $10^{-6}$ for energy equation and $10^{-4}$ for other equations.
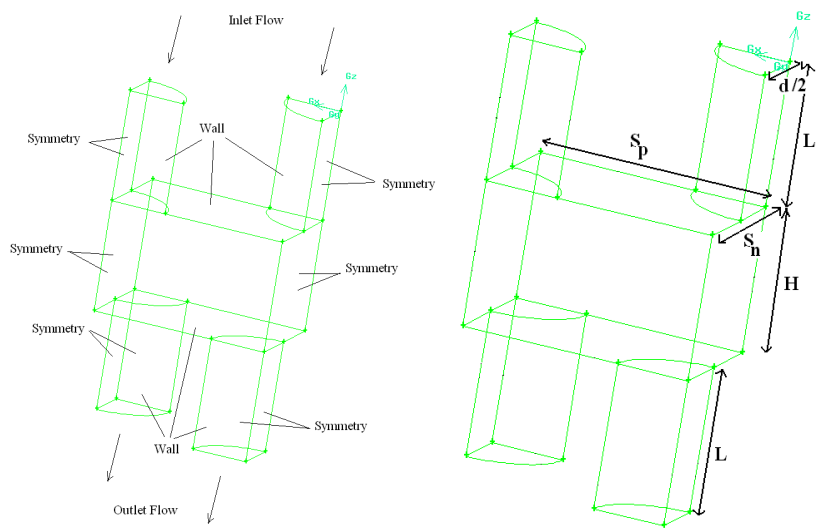

Figure 2: Computational domain, boundaries, and geometrical parameters.

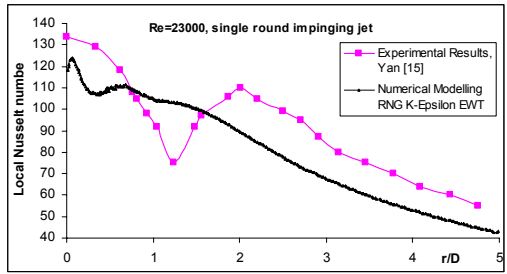

Figure 3: Comparison of numerical and experimental results for single round impinging jet.

\section{Results and discussion}

The numerical model in two dimensions was validated by Ramezanpour et al. [10]. A three dimensional computational domain of a single round nozzle impinging jet in case of $H / D=2$ and $R e=23000$ was solved and the local Nusselt number was compared in the experimental study of Yan [15] (Fig. 3). Although the results of numerical model is not satisfactory in predicting the local Nusselt number where second peak happens in the transitional location to wall jet region, however the average Nusselt number shows acceptable results. It is noted that the local trends of the Nusselt number in stagnation region depend strongly to the inlet turbulence and velocity profile (Behnia et al. [8]). 
The global heat transfer rate on the impinging and confinement plates, as well as pressure drop versus $H / D$ in different Reynolds numbers were presented in Fig. 4. The global Nusselt number is more sensitive to $H / D$ when $H / D<1$ and it increases sharper in this region by reduction of $H / D$ in both impinging and confinement plates. By increasing the $H / D$, the global Nusselt number on confinement plate reduces as the upper adverse vortexes becomes weaker near the surface, however on impinging surface it slightly increases, particularly in high Reynolds number, which may be due to weaker lower adverse vortexes and more effective impinging area. The pressure drop increases in lower $H / D$ s however this increase is sharper when $H / D<0.5$.

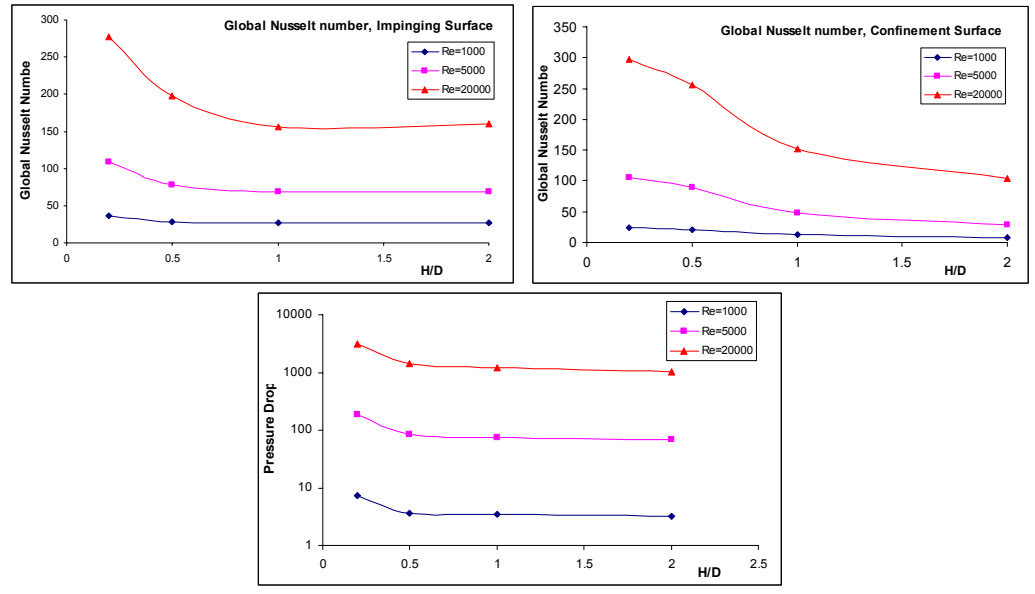

Figure 4: Global heat transfer rate and pressure drop.

The local Nusselt number in the jet to jet direction on impinging and confinement plates are shown in Fig. 5. On the impinging surface the local heat transfer rate reaches to a maximum in the stagnation point where two wall jet flows interact and at the same time there are secondary peaks due to transition point before reaching to the maximum point. This is sharper in high Reynolds number however when $R e=1000$, the local heat transfer rate almost remains constant in jet to jet interacting region. The interaction is also more effective on local heat transfer rate in lower $H / D$ s.

The trends of the local Nusselt number on confinement surface is strongly depend on $H / D$. For $H / D=1$ and 2 , the main vortexes are weak near to the confinement surface and the heat transfer rate is low and almost constant. When $H / D=0.5$, the interaction of the wall jets leads to single high momentum flow impinging to the middle of the two jets on confinement surface and therefore there is a single maximum heat transfer rate point. For $H / D=0.2$, the interaction happens in more confined space and lower adverse vortexes are stronger while upper adverse vortexes are to be shaped. Therefore the main vortex of each jet impinges to the confinement surface slightly near to the jet and two maximum local heat transfer rate occurs. The Nusselt number in the middle of the jet to jet 
distance is reached to a minimum due to weak upper vortexes forming. The small maximums detected near the jet exits are due to the weak vortexes on the edge of entering flow.
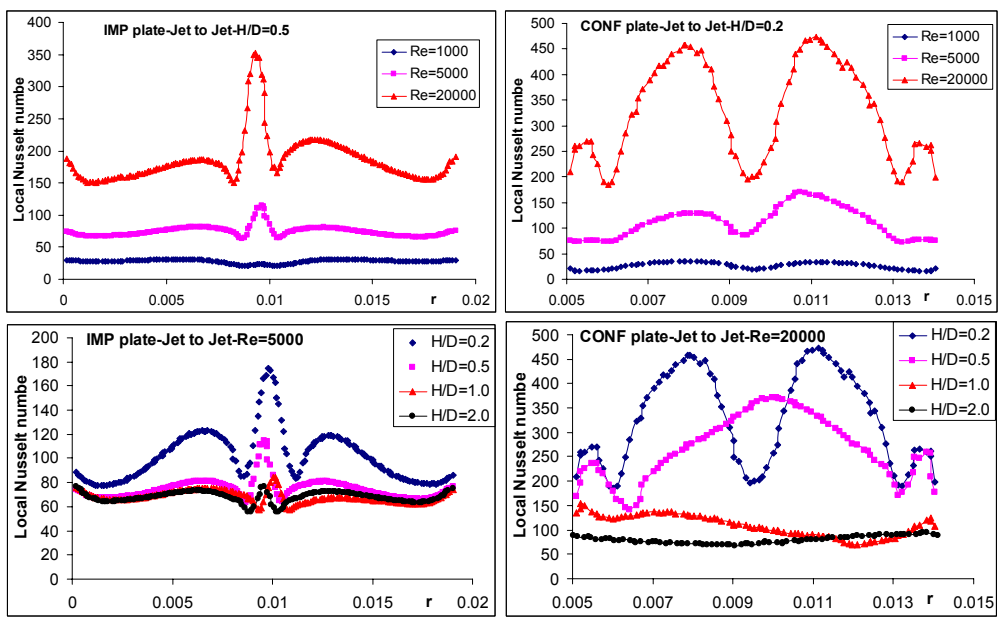

Figure 5: Local Nusselt number in jet to jet direction and on impinging and confinement surface.

Figure 6 shows velocity vectors for $H / D=0.2$ and $R e=20000$ in jet to jet section as well as symmetric boundaries in $\mathrm{S}_{\mathrm{n}}$ (shorter inlet to outlet tube distance) and $S_{p}$ (longer inlet to outlet tube distance) directions (see Fig. 2). In the jet to jet section, two main vortexes as well as lower adverse vortexes were detected while two upper adverse vortexes are to be shaped. The velocity vectors in symmetric boundaries shows influence of outlet flows on main vortexes. The main vortex on confinement surface is stronger in $\mathrm{S}_{\mathrm{n}}$ direction however the wake flow on the edge of entrance to outlet tube is weaker when distance between inlet-to-outlet tubes is longer ( $\mathrm{S}_{\mathrm{p}}$ direction). Thus one expects higher local Nusselt number on confinement plate in $S_{n}$ direction and on outlet tube in $S_{p}$ direction.
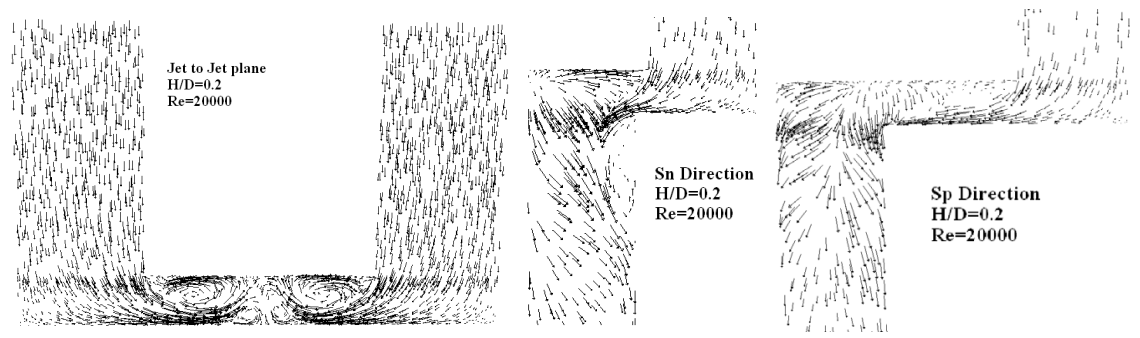

Figure 6: Velocity vectors (jet to jet, $\mathrm{Sn}$, and Sp directions). 

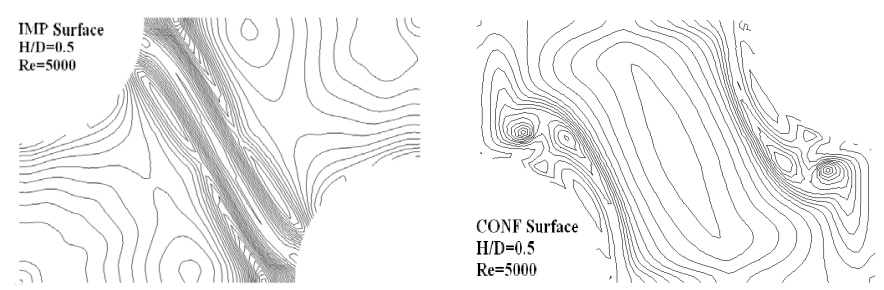

Figure 7: Temperature contours on impinging and confinement surfaces.

The temperature contours on impinging surface shows the curved maximum local Nusselt number location as well as secondary peaks in the interaction region of wall jets (Fig. 7). In $H / D=0.5$ the single maximum local Nusselt number line (almost at right angle to the jet to jet line) is seen on the confinement surface. These maximums are higher in the intersection of direct jet to jet line however they become weaker as distance from this line increases.

\section{Conclusion}

A three dimensional numerical flow and heat transfer study was conducted to investigate the characteristics of external flow of a novel patented compact heat exchanger comprising staggered array of impinging jets flowing in to opposite staggered array of tubes after impingement. The global heat transfer rate and pressure drop shows $H / D=0.5$ as an optimum design point. The local Nusselt number on impinging surface reaches a maximum with secondary maximum locations on each side however the heat transfer rate on the confinement surface depends strongly on $H / D$. The velocity vectors show different vortexes due to interaction of jets and temperature contours confirms results of local Nusselt number.

\section{References}

[1] Carcasci C., An Experimental Investigation on Air Impinging Jets Using Visualization Methods. Int. J. Thermal Science, 38, p. 808-818, 1999.

[2] Jung-Yang S., Chin-Hao H., Ming-Hong S., Impingement Cooling of a Confined Circular Air Jet. Int. J. Heat Mass Transfer, 40(6), p. 1355-1364, 1997.

[3] Beitelmal A. H., Saad M. A., Patel C. D., The Effect of Inclination on the Heat Transfer between a Flat Surface and an Impinging Two-dimensional Air Jet. Int. J. Heat Fluid Flow, 21, p. 156-163, 2000.

[4] Chin-Yuan L., Suresh V. G., Prandtl-number Effects and Generalized Correlations for Confined and Submerged Jet Impingement. Int. J. Heat Mass Transfer, 44, p. 3471-3480, 2001. 
[5] Morris G. K., Garimella S. V., Fitzgerald J. A., Flow-Field Prediction in Submerged and Confined Jet Impingement Using the Reynolds Stress Model. J. Electronic Packaging, 121, p. 255-262, 1999.

[6] Craft T. J., Graham L. J. W., Launder B. E., Impinging Jet Studies for Turbulence Model Assessment-II. An Examination of the Performance of Four Turbulence Models. Int. J. Heat Mass Transfer, 36(10), p. 26852697, 1993.

[7] Durbin P. A., On the $k-\varepsilon$ Stagnation Point Anomaly. Int. J. Heat Fluid Flow, 17, p. 89-90, 1996.

[8] Behnia M., Parneix S., Shabany Y., Durbin P. A., Numerical Study of Turbulent Heat Transfer in Confined and Unconfined Impinging Jets. Int. J. Heat Fluid Flow, 20, p. 1-9, 1999.

[9] Park T. S., Sung H. J., Development of a Near-Wall Turbulence Model and Application to Jet Impingement Heat Transfer. Int. J. Heat Fluid Flow, 22, p. 10-18, 2001.

[10] Ramezanpour A., Shirvani H., Mirzaee I., Heat Transfer of Slot Jet Impinging on an Inclined Plate, Eighth Int. Conf. on Adv. Comp. Meth. in Heat Transfer, Lisbon, Portugal, 2004.

[11] San J. Y., Lai M. D. Optimum Jet-to-Jet Spacing of Heat Transfer for Staggered Arrays of Impinging Air Jets. Int. J. Heat Mass Transfer, 44, p. 3997-4007, 2001.

[12] Su L. M., Chang S. W. Detailed Heat Transfer Measurements of Impinging Jet Arrays Issued from Grooved Surface. Int. J. Thermal Science, 41, p. 823-841, 2002.

[13] Fluent User Guide, Fluent Inc., 2005.

[14] Kadar B. A., Temperature and Concentration Profiles in Fully Turbulent Boundary Layer. Int. J. Heat Mass Transfer, 24(9), p. 1541-1544, 1981.

[15] Yan X., A preheated-wall transient method using liquid crystals for the measurement of heat transfer on external surfaces and in ducts. $\mathrm{PhD}$ Thesis, University of California, Davis, 1993. 